possible bearing on it have been obtained from observations on the spleen in an unselected series of 163 routine obstetric necropsies. In the course of this work it became obvious that there was some relation between splenic enlargement and gross hyperplasia of bone marrow, so the details about the latter are included in this analysis.

In the normal adult the spleen has a mean weight of $150 \mathrm{~g}$., with a range of from 80 to $200 \mathrm{~g}$. The observations made by Turnbull (Vaughan, 1936) on the bone marrow in normal non-pregnant patients may be briefly summarized here. His data were obtained, as in the present cases, from longitudinal section of the whole length of the femur. He found that between the ages of 15 and 20 the marrow is fully red, with specific gravity over 1000 ,

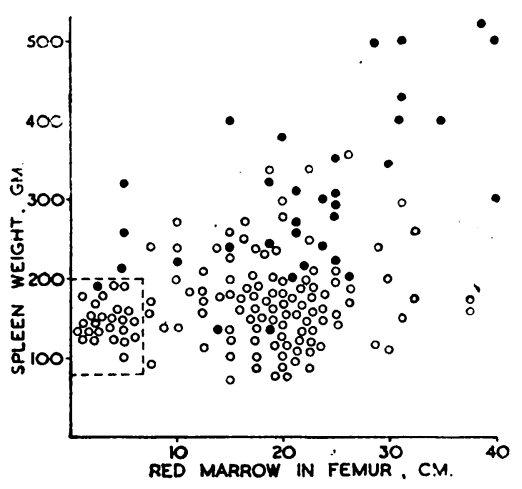

Chart showing weight of spleen and length of red marrow in femur in obstetric necropsies. $=$ Severe anaemia of pregnancy, accidental haemorrhage, or uterine sepsis and thrombophlebitis. $\mathrm{O}=$ Other clinical conditions. The panel in the left lower corner encloses the normal range in non-pregnant adults.

but in the other $40 \%$ it has a weight range of from 200 to $550 \mathrm{~g}$., so that the mean weight over the whole series is $200 \mathrm{~g}$. The red bone marrow of the femur is usually hyperplastic, though it varies from only a slight amount at the head of the bone to a complete replacement of all the yellow marrow down to the condyles. The age of the patient is without influence on the marrow hyperplasia. On the average the upper $16 \mathrm{~cm}$. is fully red marrow with a specific gravity of over 1000 , and the next $6 \mathrm{~cm}$. is partly red marrow with a specific gravity below 1000. For convenience the amount of red marrow is represented in this paper by a single figure-the length of the fully red marrow plus haif the length of the partly red marrow.

The relation between the size of the spleen and the amount of bone marrow in the present series of obstetric necropsies is shown in the above Chart. Apart from the conditions specifically mentioned below, there were no diseases of the type which give rise to enlargement of the spleen and bone marrow in ordinary pathologye.g., leukaemias, Banti's disease, tropical diseases, etc. The individual cases have been analysed in a search for significant factors. This analysis shows that in obstetric patients the following three clinical conditions are commonly associated with enlargement of the spleen and red marrow.

1. Severe Anaemia of Pregnancy.-In 9 cases the mean weight of the spleen was $360 \mathrm{~g}$. and the mean length of the red marrow of the femur was $29 \mathrm{~cm}$. It is possible that some of the other patients with enlargement of the spleen and red marrow were anaemic but were not noted as such in the clinical records ; many of them were obstetric emergencies and had not been examined haematologically.

2. Accidental Haemorrhage of Abruptio Type.-In 10 cases the mean weight of the spleen was $275 \mathrm{~g}$. and the mean length of the marrow $23 \mathrm{~cm}$. Eclampsia and the other toxaemias of pregnancy and shock or haemorrhage did not appear to be associated with these pathological increases.

3. Puerperal Thrombophlebitis or Gross Septic Endometritis. - In 15 cases the mean weight of the spleen was $290 \mathrm{~g}$. and the mean length of the marrow $18 \mathrm{~cm}$. On the other hand, general peritonitis, empyema, pneumonia, pyelonephritis, and chronic valvular disease of the heart were not associated with enlargement of the spleen or marrow.

In the Chart these three clinical conditions are differentiated from the others. It will be seen that they account for most of the large spleens and red marrows, though they do not invariably cause such enlargement. Most of the small spleens in this group were in cases of septic endometritis without thrombophlebitis.

Two other findings from the analysis may be mentioned: (a) the pathological changes were not related to the age or parity of the patients, nor to whether the patient was still pregnant or had been delivered; and $(b)$ there was some association between the enlargement of the spleen and the duration of pregnancy. The higher figures in the

\begin{tabular}{|c|c|c|c|}
\hline \multirow{2}{*}{ Gestation } & \multirow{2}{*}{ No. of Cases } & \multicolumn{2}{|c|}{ Mean Size } \\
\hline & & Spleen & Marrow \\
\hline $\begin{array}{l}0-20 \text { weeks } \\
21-30 \\
31 \text { weeks" to term }\end{array}$ & $\begin{array}{r}16 \\
10 \\
137\end{array}$ & $\begin{array}{l}145 \mathrm{~g} . \\
175 \mathrm{~g} . \\
203 \mathrm{~g} .\end{array}$ & $\begin{array}{l}16.0 \mathrm{~cm} . \\
18.5 \mathrm{~cm} . \\
19.0 \mathrm{~cm} .\end{array}$ \\
\hline
\end{tabular}

cases after the thirtieth week of gestation are partly due to the inclusion in this group of most of the patients suffering from the three significant clinical complications. It is, however, worthy of note that the largest spleen in the patients before the twentieth week of gestation weighed only $190 \mathrm{~g}$.

The essential point that emerges from this analysis is that during the course of pregnancy a number of patients have considerable splenic enlargement, which develops in the second half of gestation and is usually associated either with anaemia or with accidental haemorrhage. This is presumably accompanied by some alteration of the blood supply to the spleen. There is as yet insufficient information to indicate whether or not this is related to the relatively high incidence of rupture of splenic aneurysms in late pregnancy, but this aspect should be considered in future cases of such aneurysms.

REFERENCES

Lennie, R. A., and Sheehan, H. L. (1942). J. Obstet. Gynaec. Brit. Emp., 49, 426.

Ogden, J. K.' (1948). British Medical Journal, 1, 389.

Vaughan, J. M. (1936). The Anaemias. Oxf. Med. Publ., London.

\section{CALCIFIED CYST OF SPLEEN}

\section{BY}

JOHN H. DONOVAN, F.R.C.S.Ed.

Honorary Surgeon, Camborne and Redruth Hospital

The following seems to be the seventh recorded case of calcified splenic cyst, and the second reported in this country. There is little to be found about cysts of the spleen in the textbooks. The literature of the condition was reviewed by Harmer and Chalmers (1946), and at that date 163 cases of splenic cysts of all types had been recorded. Different classifications of the types of cyst have been made, that of Fowler (1940) being the most comprehensive. For practical purposes it would seem sufficient to classify them as parasitic, true, and false. Hydatid disease accounts for approximately $2 \%$ of recorded cases. True cysts are of endothelial origin and are usually multiple-numerous daughter cysts surrounding a large mother cyst. Congenital 
polycystic spleen is occasionally found post mortem. The solitary false cyst is probably the end-result of a haematoma.

\section{Case Report}

A spinster aged 50 complained of vague abdominal pain and intermittent attacks of nausea and vomiting. During routine barium-meal examination a shadow was seen; no other abnormality was found. Of the possible diagnoses, calcified hydatid cyst of the liver was thought to be the most probable. Casoni's reaction was negative and the blood investigations were normal. The diagnosis of calcified aneurysm of the splenic artery, of which 90 cases are on record, was discounted because of the absence of bruit. The spleen was not palpable : in the other recorded cases the palpability of the spleen was stressed as a diagnostic factor.

By transthoracic approach a portion of the left ninth rib was removed under closed-circuit cyclopropane anaesthesia and the lung partially collapsed. The cyst could then be seen pressing the diaphragm upwards. On opening the diaphragm over the bulge the cyst was found to occupy the greater portion

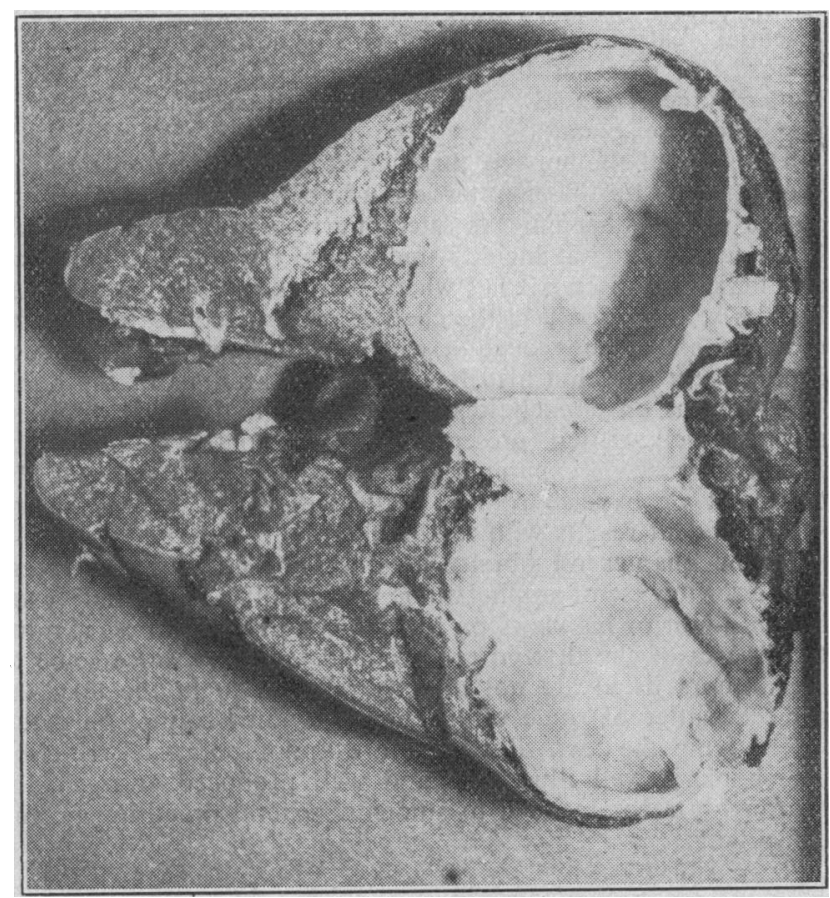

of an otherwise normal spleen (see Fig.). There were numerous adhesions, especially to the stomach, and it is assumed that these were the cause of the symptoms. Splenectomy was performed and the wound closed. Convalescence was uneventful, and the patient left hospital two weeks after operation.

\section{Discussion}

In the case recorded by Snoke (1943) the patient had been examined a few months previously for subacute cholecystitis, and no calcified shadow had then been visible on the radiographs. When the calcified cyst was removed and investigated no lining membrane was found, and Snoke considered it to be a calcified haematoma. The cholecystogram had revealed impaired liver function, and the haematoma probably resulted from the consequent hypoprothrombinaemia. Since calcification has been demonstrated in haematomata within twelve days of their appearance this seems a very reasonable hypothesis. In my case no lining was found; the cyst contained a few drachms of serum. It is interesting to note that two weeks after the patient left hospital she had a transient attack of jaundice, and therefore probably an associated hypoprothrombinaemia. I consider that this also was a calcified haematoma. Bazeley (1948) presents a similar case for diagnosis, which at the time of writing has not been confirmed by operation. In the discussion on his case the point is made that trauma may play a part in the aetiology. If there is a preceding prothrombin deficiency, trauma may well be a precipitating factor. No history of trauma could be elicited in my case.

\section{Summary}

A case of calcified cyst of the spleen is recorded.

Calcified shadows discovered by the radiologist in the left upper quadrant of the abdomen may be hydatid disease of the liver or spleen; calcified haematoma of spleen; or calcified aneurysm of the splenic artery, of which 90 cases are recorded and which are accompanied by bruit. Calcified tuberculoma of the spleen may occur.

Cysts of the spleen are not necessarily palpable.

REFERENCES

Bazeley, R. W. (1948). Proc. R. Soc. Med., 41, 377

Fowler, R. H. (1940). Int. Abstr. Surg., 70, 213.

Harman, M., and Chalmers, J. A. (1946). British Medical Journal, 1, 521 .

Snoke, P. O. (1943). Amer. J. med. Sci., 206, 726

\section{Medical Memoranda}

\section{A Case of Dracontiasis}

The following case of infestation with Dracunculus medinensis is considered worthy of record on account of the highly unusual pathological features.

\section{CASE REPORT}

An adult male African aged 26 was admitted to hospital on Nov. 1, 1944, with a diagnosis of left indirect inguinal hernia. He stated that he had had a lump in his groin for four years. He had never had any pain or discomfort.

On examination he was found to have a small indirect left inguinal hernia, which was easily reducible and which did not extend into the scrotum. No other abnormality was discovered. Blood films were negative for both malaria parasites and microfilariae. A differential white cell count showed an eosinophilia of $8 \%$.

Operation was performed on Nov. 4 through a left inguinal incision. When the hernial sac was isolated its wall was seen to be grossly thickened and fibrosed. On opening the sac it was found to contain part of a female guinea-worm. The head and about 2 in. $(5 \mathrm{~cm}$.) of the body of the worm were lying free in the lumen, the remainder being embedded in the wall of the sac on the antero-lateral aspect. The head of the worm was gently grasped in a haemostat, and by twisting the instrument slowly steady traction was maintained on the worm, which was drawn out intact. The operation was concluded in the normal manner, convalescence was uneventful, and the wound healed by first intention. The sutures were removed on the seventh day and the patient was discharged on the 21st day.

Pathology.-Although the worm closely resembled Dracunculus medinensis on superficial examination, the unusual site of infestation led to some doubt about the diagnosis. The worm was therefore carefully examined in order to make certain of the species. The overall length was $23.7 \mathrm{~cm}$. and the mean diameter $1.4 \mathrm{~mm}$. The body was an elongated cylindrical cord with a rounder anterior end and a hooked posterior end. The mouth parts and genitals were typical of Dracunculus medinensis. No male was found, and the worm did not appear to be gravid.

\section{COMMENT}

Although rather smaller than usual, the worm, as described, conformed in all other ways to the characteristics of the species Dracunculus medinensis.

Stitt (1943) states that in $1 \%$ of cases of dracontiasis the site of infestation is the scrotum. There seems to be, however, no previous record of the worm occurring in a hernial sac.

I am indebted to the Director of Medical Services, Nigeria, for permission to publish this case.

W. L. M. PERry, M.D. 